DOI: 10.12731/2227-930X-2017-3-46-63

УДК 621.865.8

\title{
РАЗРАБОТКА ИМИТАЦИОНОЙ МОДЕЛИ ПРОМЫШЛЕННОГО РОБОТА ТУР10-К С ИСПОЛЬЗОВАНИЕМ ПРОГРАММНОГО КОМПЛЕКСА «УНИВЕРСАЛЬНЫЙ МЕХАНИЗМ»
}

Чирков В.В., Шевцов А.С.

Манипуляционные роботы представляют собой сложные пространственные механические системы, имеющие пять или шесть степеней свободы, а иногда и больше. Уже только поэтому моделирование движения манипуляционных роботов даже в кинематической постановке является сложной математической задачей. Если от кинематического моделирования движения перейти $\kappa$ динамическому моделированию, учитывающему инерционные свойства объекта моделирования, аналитическое построение математической модели такого сложного объекта, как манипуляиионный робот, становится практически невозможным. Поэтому для моделирования сложных механических систем используют специальные компьютерные системы автоматизированного проектирования (САПР), так называемые САE-системы (coтputer aided engineering). Целью данной работы стоит построение имитационной модели сложной механической системы, такой как промышленный робот ТУР10-К для получения его динамических характеристик. Разработка подобных моделей позволяет сократить трудоемкость прочесса проектирования сложных систем и получить необходимыле характеристики.

Цель - разработка имитационной модели промышленного робота ТУР10-К и получение динамических характеристик механизма.

Метод или методология проведения работы: в статье использовался компьютерный метод моделирования.

Результаты: получена имитационная модель робота и его динамические характеристики. 
Область применения результатов: результатьл могут быть использованы при проектировании механических систем и различных имитационных моделей.

Ключевые слова: Манипулячуионный робот; динамическое моделирование; CAD-; CAE-системы.

\section{DEVELOPING INDUSTRIAL ROBOT SIMULATION MODEL TUR10-K USING "UNIVERSAL MECHANISM" SOFTWARE COMPLEX}

\section{Chirkov V.V., Shevtsov A.S.}

Manipulation robots are complex spatial mechanical systems having five or six degrees of freedom, and sometimes more. For this reason, modeling manipulative robots movement, even in the kinematic formulation, is a complex mathematical task. If one moves from kinematic modeling of motion to dynamic modeling then there must be taken into account the inertial properties of the modeling object. In this case, analytical constructing of such a complex object mathematical model as a manipulation robot becomes practically impossible. Therefore, special computer-aided design systems, called CAE-systems, are used for modeling complex mechanical systems. The purpose of the paper is simulation model construction of a complex mechanical system, such as the industrial robot TUR10-K, to obtain its dynamic characteristics. Developing such models makes it possible to reduce the complexity of designing complex systems process and to obtain the necessary characteristics.

Purpose. Developing the simulation model of the industrial robot TUR10-K and obtaining dynamic characteristics of the mechanism.

Methodology: the article is used a computer simulation method.

Results: There is obtained the simulation model of the robot and its dynamic characteristics.

Practical implications: the results can be used in the mechanical systems design and various simulation models.

Keywords: manipulation robot; dynamic modeling; CAD-; CAE-systems. 
Для создания 3D-модели манипуляционного робота может быть использована CAD-система KOMПАC-3D, обладающая всем необходимым инструментальным арсеналом для построения сложных 3D-моделей, включая создание сборок. Именно сборка 3D-модели манипуляционного робота потребуется для построения его имитационной модели [1-4]. Сборка 3D-модели выполняется из заранее разработанных деталей и других сборок. Основными составляющими, определяющими функциональное назначение манипуляционного робота, являются звенья. Звенья моделируются составляющими их деталями [5-7].

CAD-системы обладают всем необходимым для построения сборок 3D-моделей механизмов. Однако в нашем случае воспользуемся готовой 3D-моделью манипуляционного робота ТУР-10K, имеющего пять степеней подвижности (рис. 3). Загрузим модель в КОМПАС-3D V16. При этом файл модели преобразуется в формат данной CAD-системы. Преобразованная модель будет представлять собой единую 3D-деталь, состоящую из отдельных элементов. Выделив элементы, составляющие звенья промышленного робота, сохраним их в отдельных файлах с расширением m3d. B названии этих файлов удобно использовать нумерацию звеньев. Далее, объединяя эти файлы, выполняется сборка 3D-модели всего манипуляционного робота и формируется файл с расширением a3d (рис. 2).

Помимо геометрических изображений в файлах деталей и сборок CAD-система сохраняет информацию о так называемых массово-центровочных характеристиках 3D-модели. Эти характеристики содержат значения плотности материала, из которого изготовлены детали, их массы и координаты центра масс.

Для построения имитационной модели манипуляционного робота используем программный комплекс «Универсальный механизм», являющийся САЕ-системой [8]. Нужно запустить программy UM Input, которая содержит необходимый инструментарий для создания имитационных моделей сложных механических систем. Для переноса 3D-модели исследуемого робота в программу 
UM Input следует использовать команды меню этой программы: «Инструменты» $\rightarrow$ «Импорт из CAD» $\rightarrow$ «КOMПАC-3D». Ocoбенность реализации импорта данных в данном программном комплексе требует, чтобы соответствующая CAD-система была инсталлирована на одном компьютере с UM. При выполнении команды импорта данных UM проверяет, активно ли приложение соответствующей CAD-системы, в данном случае KOMПАC-3D, и если не активно, запускает это приложение и загружает выбранную сборку. При этом конвертируются графические образы и массово-центровочные характеристики каждой детали, входящей в сборку 3D-модели. После конвертации создаётся новый UM-объект. Рассмотрим создание имитационной модели манипуляционного робота в UM.

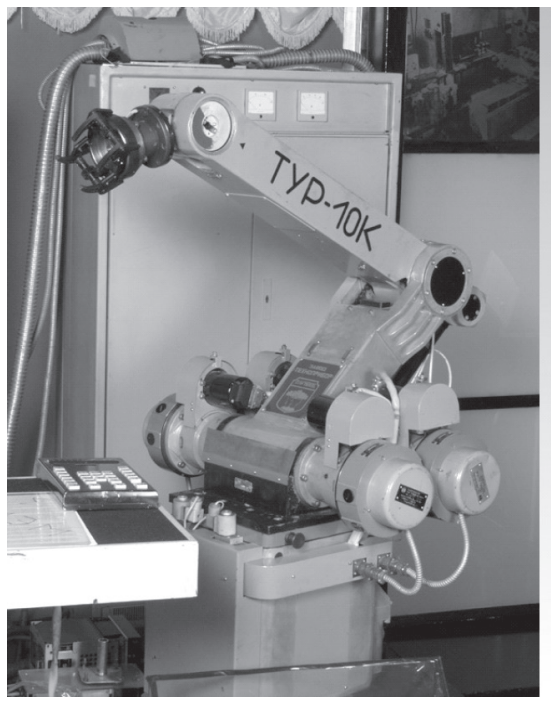

Рис. 1. ТУР-10K

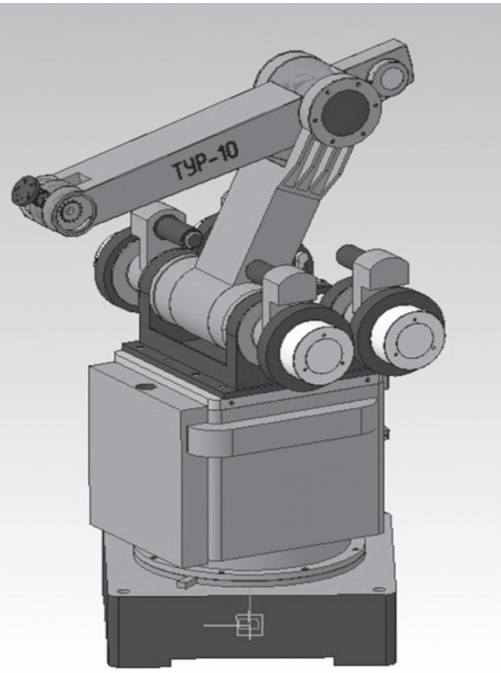

Рис. 2. 3D-модель робота ТУР-10K

Созданный при конвертации UM-объект будет содержать составляющие его тела, соответствующие деталям исходной сборки. Для создания имитационной модели необходимо все тела связать между собой шарнирами. 
При конвертации 3D-модели промышленного робота ТУР-10 создаётся объект, состоящий из шести тел Z0-Z5, - по числу деталей, соответствующих звеньям робота от 0 до 5. Для введения в объект шарниров используем команды меню программы UM Input: «Добавить» $\rightarrow$ «Шарниры» $\rightarrow$ «Вращательный». Исключение составит тело Z0 - основание. Это тело свяжем с неподвижным основанием шарниром с нулевой степенью свободы. Данный шарнир вводится набором команд меню: «Добавить» $\rightarrow$ «Шарниры» $\rightarrow$ «6 ст. свободы». При создании любого шарнира необходимо указывать два тела, связываемые этим шарниром (тело 1 и тело 2). Для первого введенного шарнира тело 1 - Base0, а тело 2 - Z0, при этом создаётся шарнир jBase $0->0$. В этом шарнире необходимо отключить все поступательные и вращательные степени свободы, образовав тем самым неподвижное соединение. Первое звено Z1 свяжем со стойкой Z0 вращательным шарниром jZ1 (рис. 3). На рисунке шарнир показан в виде красного цилиндра. При назначении шарнира важно задать его положение относительно осей, при этом будет изменяться и положение красного цилиндра, при помощи которого обозначается центр шарнира и его ориентация относительно системы координат и других деталей механизма. Такое обозначение является очень удобным и наглядным решением, которое упрощает построение имитационной модели. Второе звено Z2 свяжем с первым звеном Z1 аналогичным шарниром jZ2 (рис. 4). Особенность связки этих двух звеньев и всех последующих заключается в том, что их центры шарниров находятся в каких-то точках, имеющих свои координаты в базовой системе (системе координат, связанной с первым звеном). Для построения более точной имитационной модели необходимо знать координаты центра шарниров в базовой системе и направление их осей. Наиболее точные значения для шарниров можно получить из 3D модели построенной в CAD-системе (нашем случае это «КОМПАС-3D»), либо измерив сам робот при помощи различных измерительных приборов, например, координатно-измерительных машин. Остальные звенья соединяются последовательно аналогичным образом (рис. 5-7). 


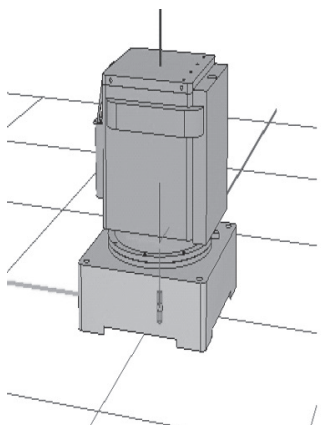

Рис. 3. Шарнир jZ1

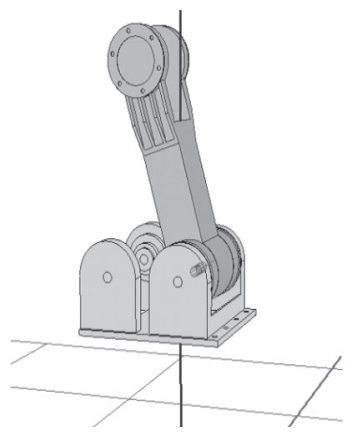

Рис. 4. Шарнир jZ2

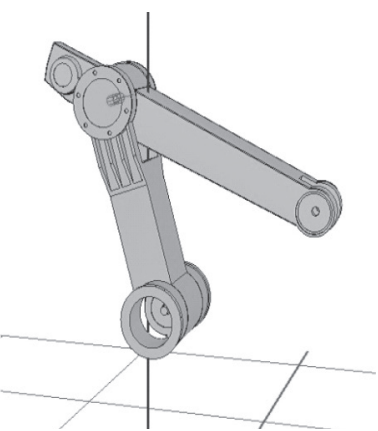

Рис. 5. Шарнир jZ3

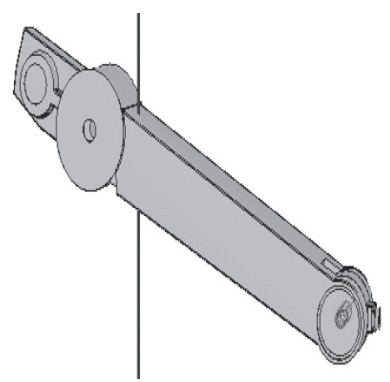

Рис. 6. Шарнир jZ4

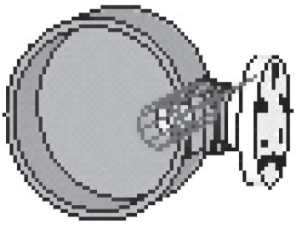

Рис. 7. Шарнир jZ5

Промышленный робот ТУР-10К имеет редкую компоновку, она заключается в том, что приводы третьего и последующих звеньев располагаются на втором звене, а движения передаются при помощи системы рычагов. Для разработки полноценной имитационной модели необходимо также построить схему рычагов и связать их шарнирами. Делается это аналогичным образом, как и в случае со звеньями робота. Система рычагов представляет цепь звеньев (рычагов) которые соединяют выходной вал редуктора с конкретным звеном робота (рис. 8). В реальном механизме выходной вал электродвигателя присоединен к входному валу редуктора, который передает вращающий момент на систему рычагов. Для построения имитационной модели не требуется такой детальной проработки механизма, поэтому двигатель и редуктор выполнены как одна целая деталь. 


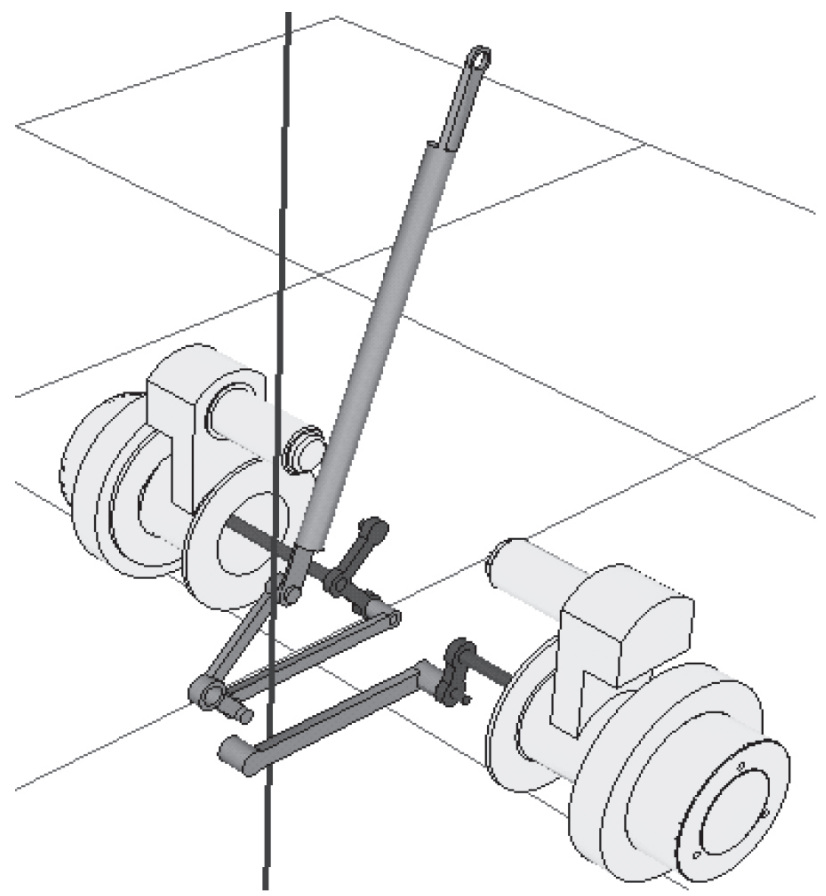

Рис. 8. Шарнирные параллелограммы

Промышленный робот ТУР-10К имеет две такие системы рычагов для управления третьим и четвертым звеньями. Системы рычагов для управления третьим звеном имеет более простую схему и меньшее количество элементов (рис. 8). Она состоит из рычага, который соединен с редуктором и из еще одного который соединяет первый рычаг и третье звено робота. Рычаг, который соединен с редуктором, передает вращательный момент от редуктора далее по цепи, в дальнейшем при моделировании движения робота для управления третьим звеном мы будем задавать законы движения для этого рычага. Процесс построения имитационной модели рычагов для управления третьим звеном представляет собой последовательность действий по привязке рычагов друг к другу при помощи шарниров (рис. 9-11). 


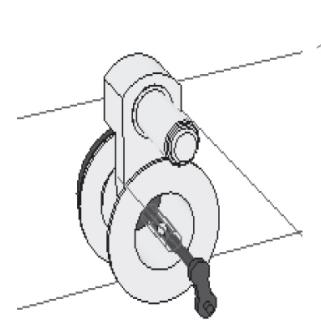

Рис. 9.

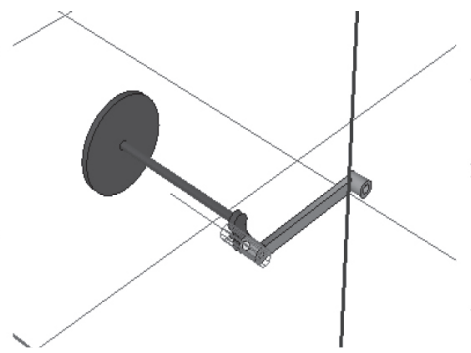

Рис. 10.

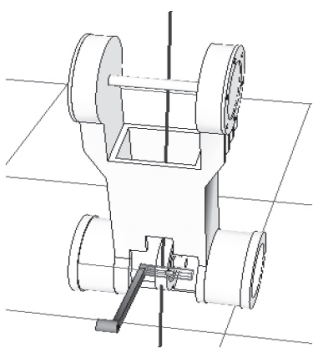

Рис. 11.

Шарнир редуктор-рычаг Промежуточный шарнир Шарнир рычаг-плечо

Система рычагов, управляющая четвертым звеном робота, имеет более сложную структуру и большее количество элементов. Она так же имеет рычаг соединяющий вал редуктора с остальной цепочкой рычагов, этому рычагу в дальнейшем будем задавать законы движения для управления четвертым звеном робота. Дальнейшее моделирование заключается в том, чтобы связать все рычаги последовательно шарнирами (рис. 12-17).

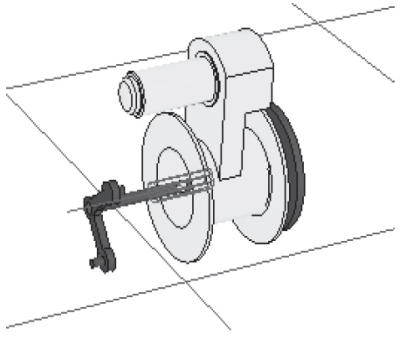

Рис. 12.

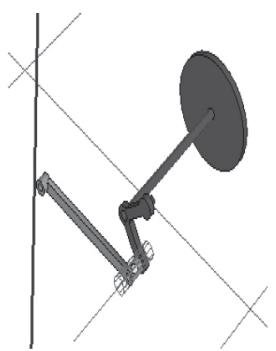

Рис. 13.

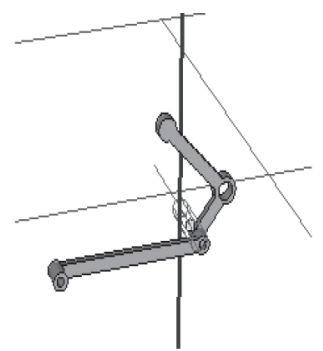

Рис. 14.

Шарнир редуктор-рычаг Промежуточный шарнир Промежуточный шарнир

Следующим шагом формирования имитационной модели манипуляционного робота в форме UM-объекта является создание силовых элементов [9-10]. Раздел меню «Добавить» программы UM Input содержит перечень реализованных в ней силовых элементов: «Биполярные силы», «Скалярные моменты», «Линейные силы», «Контактные силы», «Т - силы», «Специальные силы». Выбор необходимых силовых элементов зависит от постановки 
конкретной прикладной задачи. С целью упрощения излагаемого материала из внешних сил, действующих на манипуляционную систему моделируемого робота, будем учитывать только силы тяжести, поэтому использовать в модели специальные силовые элементы не будем.

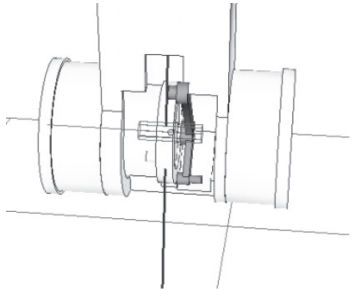

Рис. 15.

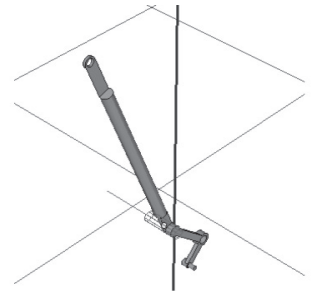

Рис. 16.

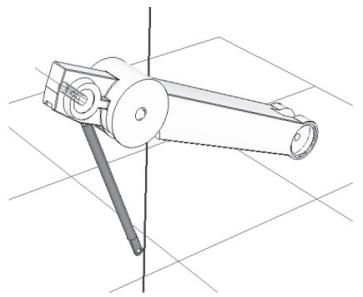

Рис. 17.

Шарнир на плече $\quad$ Промежуточный шарнир Шарнир рычаг-локоть

Для моделирования движения манипуляционного робота ТУР$10 K$ с использованием разработанной имитационной модели воспользуемся реализованной в программе UM Input возможностью задания функции времени для шарнирной координаты. В программе предусмотрено задание нескольких типов таких функций: «Выражение», «Функция», «Расписание», «Файл», «Кривая». Выберем возможность задать функцию времени выражением. Для каждого шарнира зададим выражение wi t, где wi (i=1...5) - переменные, инициализируемые в специальном окне программы, имеющие смысл относительной угловой скорости между соответствующими звеньями (телами) в данном шарнире [11-13]. Так, для первого шарнира jZ1 выражение будет иметь вид «w1*t». Значение переменной w1 необходимо задать в специальном окне, открывающемся после завершения ввода выражения функции времени.

После завершения формирования UM-объекта он сохраняется в файле input.dat, который необходимо поместить в специальную папку (каталог) с именем объекта, например, /TUR10K. Moделирование движения исследуемого объекта осуществляется в программе UM Simulation, которая может быть запущена непосредственно из программы UM Input. В программе UM Simulation 
реализован большой набор инструментов для проведения анализа движения исследуемого объекта. Например, можно вывести на рабочий стол компьютера «Анимационное окно», в котором будет отражена анимация движения исследуемого объекта, а также «Графическое окно» для построения графиков переменных, созданных исследователем. Переменные могут быть созданы мастером переменных через меню программы: «Инструменты» $\rightarrow$ «Мастер переменных».

Задав траекторию движения промышленного робота и запустив программу на выполнение, с временем интегрирования равным $6 \mathrm{c}$. Получили результаты моделирования, представленные на рис. 1822. Мы смогли получить основные параметры.

Мы получили графическое изображение движения робота и его траекторию (красная линия) (рис. 18).

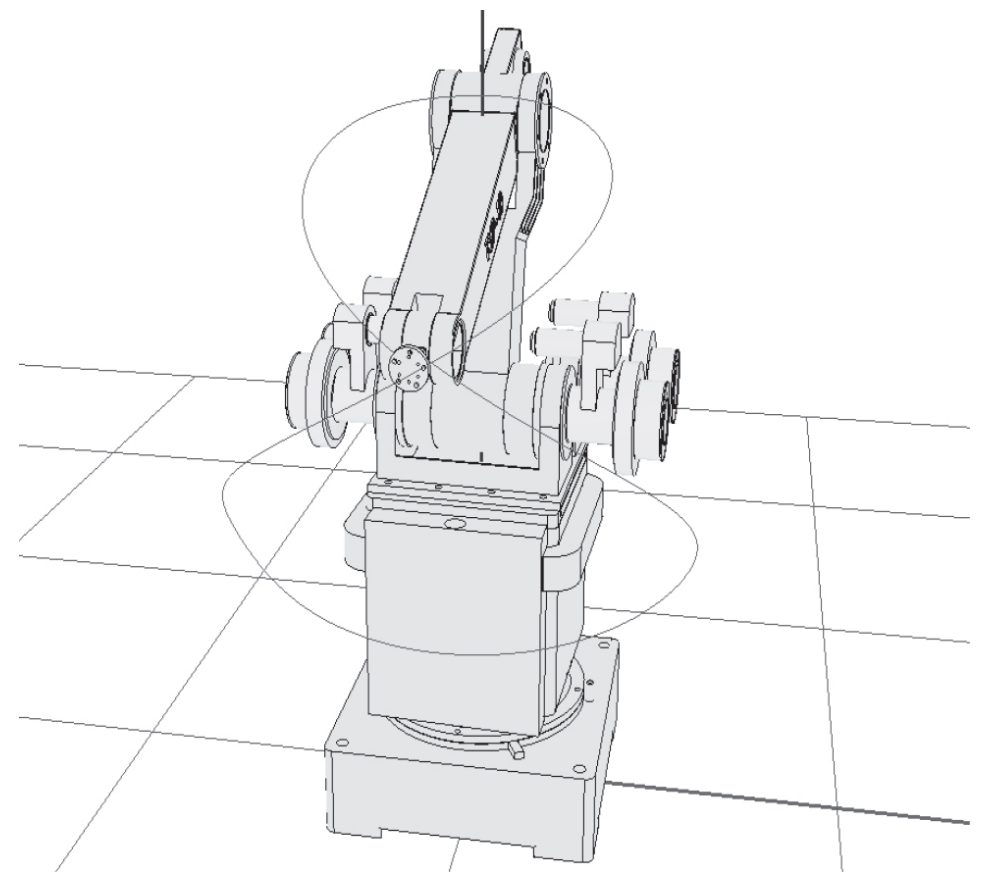

Рис. 18. Траектория движения 
Построенная имитационная модель промышленного робота ТУР10-К позволила получить нам моменты силы развиваемые в шарнирах робота при выполнении заданного движения «Восьмерка». В качестве переменных рассмотрели реакции (моменты) jRMm(jZ1), jRMm(jZ2), jRMm(jZ3), jRMm(jZ4) и jRMm(jZ5), возникающие в шарнирах jZ1- jZ5 при выполнении движения по заданным функциям времени (рис. 19). Полученные данные могут быть использованы при проектировки различных модификаций промышленных роботов на базе конструкционных решений робота ТУР10-К. Так как мы знаем значения моментов, необходимые для выполнения некоторого движения, мы можем подбирать оборудование с характеристиками пропорциональными полученным данным.

Посмотрев на полученный график шарнирных моментов, мы можем понять, что при движении без груза наиболее нагружены второй и третий шарниры.

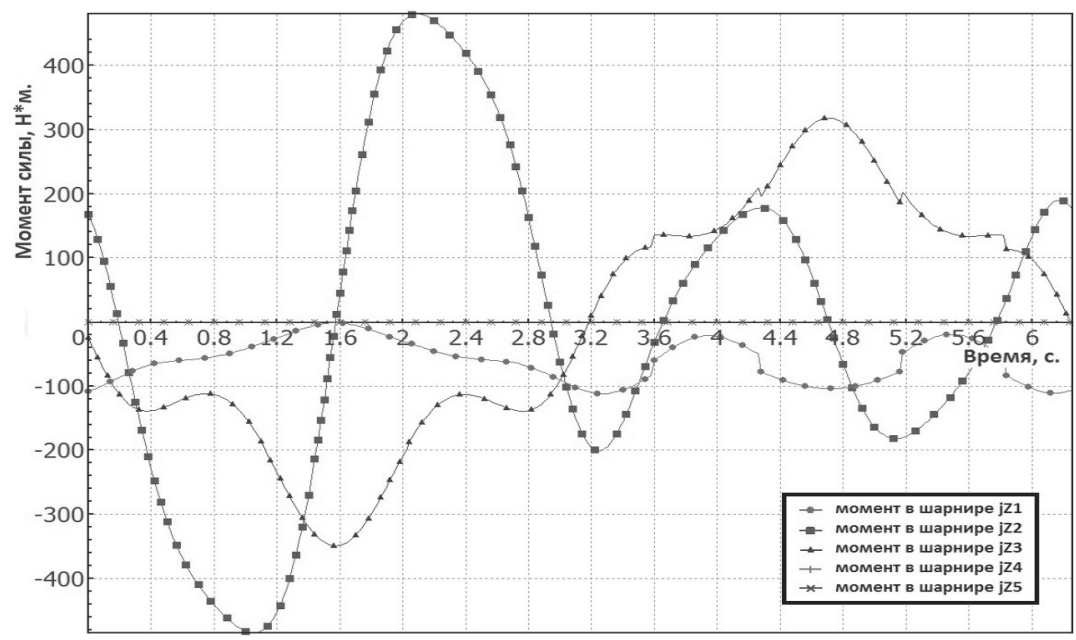

Рис. 19. Шарнирные моменты

Также были получены проекции скорости v:x(Z5), v:y(Z5) и $v: z(Z 5)$ характерной точки пятого звена (тело Z5) в базовой (неподвижной системе координат связанной с первым звеном (рис. 20). 
Из полученных графиков мы можем понять с какими скоростями движется последнее звено робота, выполняя заданное движение «Восьмерка». Это позволяет проанализировать движение робота и судить о его характеристиках исходя из полученных скоростей в отдельные промежутки времени [14].

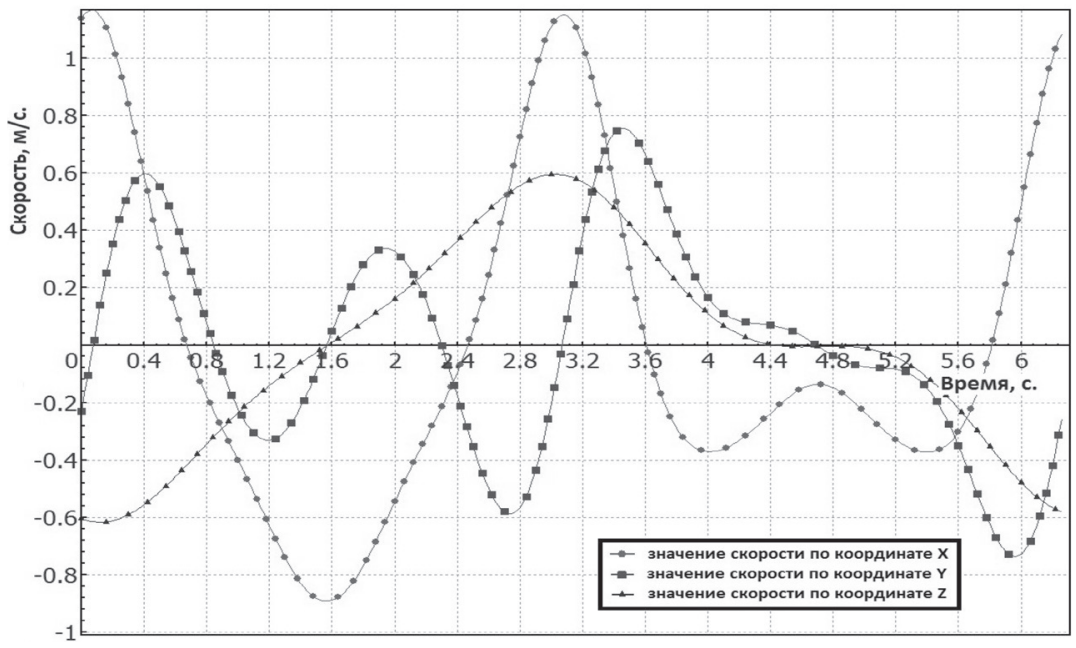

Рис. 20. Скорости точки последнего звена

Графики изменения ускорений данной точки относительно базовой системы координат (рис. 21). Из полученных графиков мы можем понять с какими ускорениями движется последнее звено робота выполняя заданное движение «Восьмерка». Это позволяет проанализировать движение робота и судить о его характеристиках исходя из полученных ускорений в отдельные промежутки времени.

И координаты характерной точки последнего звена в каждый момент времени по осям х,y и z базовой системы координат (рис. 22). Из полученных графиков мы можем получить координаты последнего звена в каждый момент времени выполняя заданное движение «Восьмерка». Это позволяет проанализировать движение робота и судить о его характеристиках исходя из полученных значений координат в отдельные промежутки времени. 


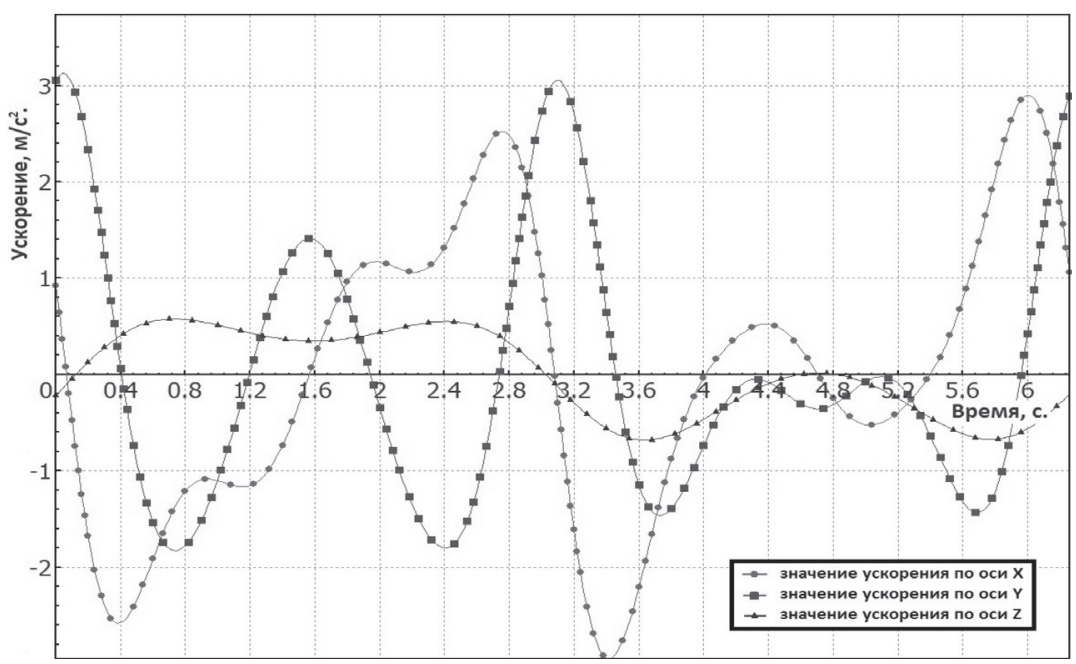

Рис. 21. Ускорения точки последнего звена

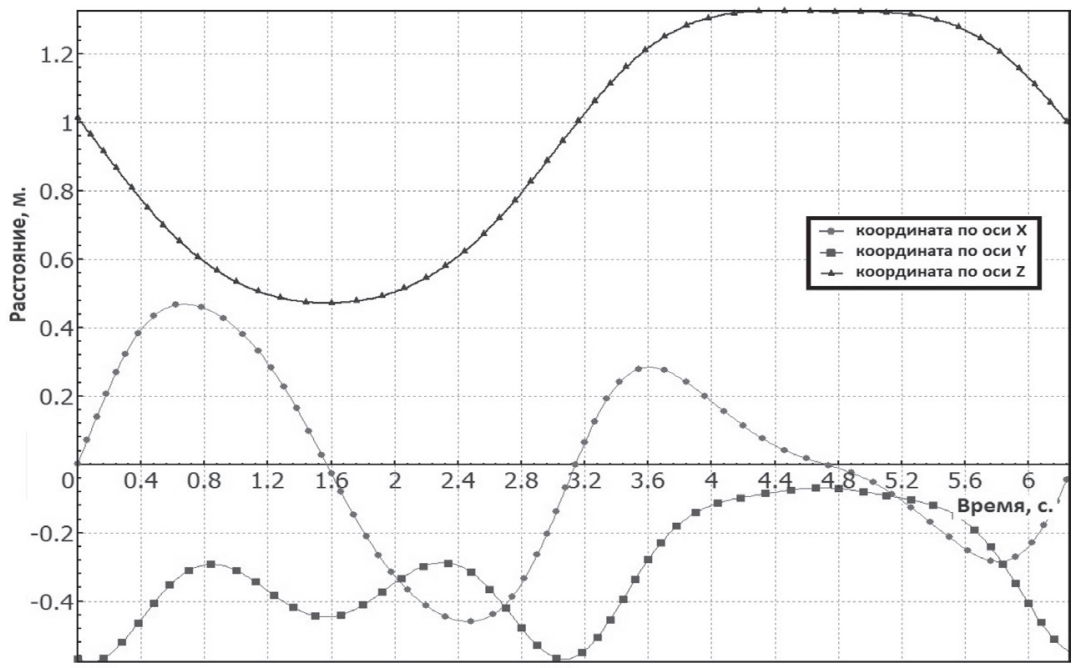

Рис. 22. Координаты точки последнего звена

В данной работе рассмотрено решение обратной задачи динамики для манипуляционной системы промышленного робота 
ТУР10К. Задача состоит в определении обобщённых сил, в данном случае шарнирных моментов, по обобщённым координатам, скоростям и ускорениям, заданным функциями времени для каждого из шарниров созданной модели [15]. Аналогично может быть рассмотрено решение прямой задачи динамики. Для этого вместо использованных функций времени для каждого шарнира должны быть заданы шарнирные моменты. Такая возможность в программе UM Input имеется.

\section{Заключение}

Возможности программного комплекса «Универсальный механизм» позволяют помимо решения задач кинематики и динамики создавать системы управления, реализующие законы управления для создаваемых имитационных моделей. Также возможно совместное моделирование нескольких объектов, что позволяет создавать имитационные модели, например, для робототехнических комплексов, включающих несколько промышленных роботов и обслуживаемое ими технологическое и вспомогательное оборудование (станки, лотки, конвейеры и т.д.).

\section{Список литературы}

1. Крахмалев О.Н. Математическое моделирование динамики манипуляционных систем промышленных роботов и кранов-манипуляторов: монография. Брянск: Изд-во БГТУ, 2012. С. 200.

2. Крахмалев О.Н. Методика анализа влияния сил инерции на динамику манипуляционных роботов // Теория механизмов и машин. 2012. T. 10, № 20. C. 41-53.

3. Крахмалев О.Н., Блейшмидт Л.И. Определение динамической точности манипуляционных систем роботов с упругими шарнирами // Проблемы машиностроения и надежности машин. 2014. № 1. C. 29-36.

4. Крахмалев О.Н., Петрешин Д.И. Исследование движения манипуляционных роботов на основе анализа параметров инерции // Мехатроника, автоматизация, управление. 2014. № 10. С. 3-6. 
5. Крахмалев О.Н., Петрешин Д.И. Коррекция интегральных отклонений движения исполнительных механизмов промышленных роботов и многокоординатных станков // Мехатроника, автоматизация, управление. 2015. Т. 16, № 7. С. 491-496.

6. Крахмалев О.Н., Петрешин Д.И., Федонин О.Н. Метод построения геометрических моделей манипуляционных систем промышленных роботов и многокоординатных станков // Наука и образование. 2015. № 5(72). C. 34.

7. Крахмалев О.Н., Петрешин Д.И., Федонин О.Н. Методика параметризации геометрических (математических) моделей манипуляционных систем промышленных роботов и многокоординатных станков // Наука и образование. 2015. № 5(72). С. 35.

8. Крахмалев О.Н., Петрешин Д.И., Федонин О.Н. Методика параметризации геометрических (математических) моделей манипуляционных систем промышленных роботов и многокоординатных станков // Наука и образование. 2015. № 5(72). С. 36.

9. http:// www.umlab.ru.

10. Krakhmalev O.N., Petreshin D.I., Fedonin O.N. Provision of controlled motion accuracy of industrial robots and multiaxis machines by the method of integrated deviations correction // IOP Conference Series: Materials Science and Engineering Cep. "International Conference on Mechanical Engineering, Automation and Control Systems 2015, MEACS 2015”, 2016. 012067. DOI: 10.1088/1757899X/124/1/012067

11. Krakhmalev O.N. Mathematical model manipulator robots // International Journal of Advanced Studies. 2015. T. 5. № 4. C. 31-35. DOI: 10.12731/2227-930X-2015-4-4

12. Krakhmalev O.N., Bleyshmidt L.I. Determination of dynamic accuracy of manipulation systems of robots with elastic hinges // Journal of Machinery Manufacture and Reliability. 2014. T. 43. № 1. C. 22-28.

13. Krakhmalev O.N. Simulation movements of robots // Актуальные направления фундаментальных и прикладных исследований Материалы VII международной научно-практической конференции. н.и. ц. «Академический». 2015. С. 151-153. 
14. Rytov M.Yu., Spichyack S.A., Fedorov V.P., Petreshin D.I. The model and control methods of access to information and technology resources of automated control systems in water supply industry // Journal of Physics: Conference Series. 2017. T. 803. № 1. C. 012132.

15.Petreshin D.I., Handozhko A.V., Fedonin O.N. An automated control system for machinery parts machining // IOP Conference Series: Materials Science and Engineering Cep. "International Conference on Mechanical Engineering, Automation and Control Systems 2015, MEACS 2015”, 2016. 012024. DOI: 10.1088/1757-899X/124/1/012024

\section{References}

1. Krakhmalev O. Matematicheskoye modelirovaniye dinamiki manipulyatsionnykh sistem promyshlennykh robotov i kranov-manipulyatorov: monografiya [Mathematical modeling of dynamics of manipulation systems of industrial robots and cranes-manipulators: monograph]. Bryansk, BSTU Publ., 2012, 200 p.

2. Krakhmalev O. Teoriya mekhanizmov i mashin, 2012, vol. 10, no 20, pp. 41-53.

3. Krakhmalev O., Bleishmidt L. Problemy mashinostroyeniya i nadezhnosti mashin, 2014, no. 1, pp. 29-36.

4. Krakhmalev O., Petreshin D. Mekhatronika, avtomatizatsiya, upravleniye, 2014, no. 10, pp. 3-6.

5. Krakhmalev O., Petreshin D. Mekhatronika, avtomatizatsiya, upravleniye, 2015, vol. 16, no. 7, pp. 491-496.

6. Krakhmalev O.N., Petreshin D.I., Fedonin O.N. Nauka i obrazovaniye, 2015, no. 5 (72), 34 p.

7. Krakhmalev O.N., Petreshin D.I., Fedonin O.N. Nauka i obrazovaniye, 2015, no. 5 (72), 35 p.

8. Krakhmalev O.N., Petreshin D.I., Fedonin O.N. Nauka i obrazovaniye, 2015, no. 5 (72), 36 p.

9. http://www.umlab.ru.

10. Krakhmalev O.N., Petreshin D.I., Fedonin O.N. Provision of controlled motion accuracy of industrial robots and multiaxis machines by the method of integrated deviations correction. IOP Conference Series: 
Materials Science and Engineering Cep. "International Conference on Mechanical Engineering, Automation and Control Systems 2015, MEACS 2015”, 2016. 012067. DOI: 10.1088/1757-899X/124/1/012067

11. Krakhmalev O.N. Mathematical model manipulator robots. International Journal of Advanced Studies. 2015.V. 5. № 4. C. 31-35. DOI: 10.12731/2227-930X-2015-4-4

12. Krakhmalev O.N., Bleyshmidt L.I. Determination of dynamic accuracy of manipulation systems of robots with elastic hinges. Journal of Machinery Manufacture and Reliability. 2014. V. 43. № 1. C. 22-28.

13. Krakhmalev O.N. SIMULATION MOVEMENTS OF ROBOTS Aktual'nyye napravleniya fundamental'nykh i prikladnykh issledovaniy Materialy VII mezhdunarodnoy nauchno-prakticheskoy konferentsii . n.-i. ts. «Akademicheskiy». [Actual directions of fundamental and applied research. Materials of the VII International Scientific and Practical Conference. Scientific and Publishing Center "Academic"]. 2015, pp. 151-153.

14. Rytov M.Yu., Spichyack S.A., Fedorov V.P., Petreshin D.I. The model and control methods of access to information and technology resources of automated control systems in water supply industry. Journal of Physics: Conference Series. 2017. T. 803. № 1. C. 012132.

15.Petreshin D.I., Handozhko A.V., Fedonin O.N. An automated control system for machinery parts machining. IOP Conference Series: Materials Science and Engineering. Cep. "International Conference on Mechanical Engineering, Automation and Control Systems 2015, MEACS 2015”, 2016. 012024. DOI: 10.1088/1757-899X/124/1/012024

\section{ДАННЫЕ ОБ АВТОРАХ}

Чирков Вадим Владимирович, студент 4 курса, бакалавриат

Брянский государственный технический университет 50 лет Октября бульвар, 7, г. Брянск, Брянская область, 241035, Российская Федерация vadimc75@gmail.com

Шевцов Александр Сергеевич, студент 4 курса, бакалавриат Брянский государственный технический университет 
50 лет Октября бульвар, 7, г. Брянск, Брянская область, 241035, Российская Федерачия

alex-s97@mail.ru

\section{DATA ABOUT THE AUTHORS}

Chirkov Vadim Vladimirovich, 4-th Year Student, Bachelor's Degree Bryansk State Technical University

7, 50 years of October Boulevard, Bryansk, Bryansk region, 241035, Russian Federation

vadimc75@gmail.com

SPIN-code: 4581-7712

Shevtsov Aleksandr Sergeyevich, 4-th Year Student, Bachelor's Degree

Bryansk State Technical University

7, 50 years of October Boulevard, Bryansk, Bryansk region, 241035, Russian Federation

alex-s97@mail.ru

SPIN-code: 8161-4953 